Journal of Nonlinear Mathematical Physics

1998, V.5, N 3, 331-348.

ARTICLE

\title{
Some Homogenization and Corrector Results for Nonlinear Monotone Operators
}

\author{
Peter WALL \\ Department of Mathematics, Luleå University of Technology, SE-971 87 Luleå, Sweden
}

Received March 5, 1998; Accepted May 9, 1998

\begin{abstract}
This paper deals with the limit behaviour of the solutions of quasi-linear equations of the form $-\operatorname{div}\left(a\left(x, x / \varepsilon_{h}, D u_{h}\right)\right)=f_{h}$ on $\Omega$ with Dirichlet boundary conditions. The sequence $\left(\varepsilon_{h}\right)$ tends to 0 and the map $a(x, y, \xi)$ is periodic in $y$, monotone in $\xi$ and satisfies suitable continuity conditions. It is proved that $u_{h} \rightarrow u$ weakly in $H_{0}^{1,2}(\Omega)$, where $u$ is the solution of a homogenized problem $-\operatorname{div}(b(x, D u))=f$ on $\Omega$. We also prove some corrector results, i.e. we find $\left(P_{h}\right)$ such that $D u_{h}-P_{h}(D u) \rightarrow 0$ in $L^{2}\left(\Omega, R^{n}\right)$.
\end{abstract}

\section{Introduction}

In mathematical models of microscopically non-homogeneous media various local characteristics are usually described by functions of the form $a\left(x / \varepsilon_{h}\right)$ where $\varepsilon_{h}>0$ is a small parameter. The function $a(x)$ can be periodic or belong to some other specific class. To compute the properties of a micro non-homogeneous medium is an extremely difficult task since the coefficients are rapidly oscillating functions. Therefore, it is necessary to apply asymptotic analysis to the problems of micro non-homogeneous media, which immediately leads to the concept of homogenization. When the parameter $\varepsilon_{h}$ is very small the medium will act as a homogeneous medium. To characterize this homogeneous medium is one of the main tasks in the homogenization theory. For more information concerning the homogenization theory the reader is referred to [1], [8] and [10]. In this paper we consider the homogenization problem for monotone operators and the local behavior of the solutions. Monotone operators are very important in the study of nonlinear partial differential equations. The problem we study here can be used to model different nonlinear stationary conservation laws, e.g. stationary temperature distribution. For a more detailed discussion concerning different applications see [13].

We will study the limit behavior of the sequence of solutions $\left(u_{h}\right)$ to the Dirichlet boundary value problem

$$
\left\{\begin{array}{l}
-\operatorname{div}\left(a\left(x, \frac{x}{\varepsilon_{h}}, D u_{h}\right)\right)=f_{h} \quad \text { on } \quad \Omega, \\
u_{h} \in H_{0}^{1,2}(\Omega),
\end{array}\right.
$$

Copyright (c) 1998 by P. Wall 
where $f_{h} \rightarrow f$ in $H^{-1,2}(\Omega)$ and $\varepsilon_{h} \rightarrow 0$. Moreover, the map $a(x, y, \xi)$ is defined on $\Omega \times R^{n} \times R^{n}$ and is assumed to be periodic in $y$, uniformly Lipschitz continuous in $\xi$ and uniformly monotone in $\xi$. We also need some continuity restriction on $a(\cdot, y, \xi)$. We will consider two different cases, namely when $a(x, y, \xi)$ is of the form

$$
a(x, y, \xi)=\sum_{i=1}^{N} \chi_{\Omega_{i}}(x) a_{i}(y, \xi),
$$

or when $a(x, y, \xi)$ satisfies that

$$
\left|a\left(x_{1}, y, \xi\right)-a\left(x_{2}, y, \xi\right)\right|^{2} \leq \omega\left(\left|x_{1}-x_{2}\right|\right)|\xi|^{2},
$$

where $\omega: R \rightarrow R$ is continuous, increasing and $\omega(0)=0$. In both cases we will prove that $u_{h} \rightarrow u$ weakly in $H^{1,2}(\Omega)$ and that $u$ is the solution of the homogenized problem

$$
\left\{\begin{array}{l}
-\operatorname{div}(b(x, D u))=f \quad \text { on } \quad \Omega, \\
u \in H_{0}^{1,2}(\Omega) .
\end{array}\right.
$$

We will prove that the operator $b$ has the same structure properties as $a$ and is given by

$$
b(x, \xi)=\int_{Y} a\left(x, y, \xi+D v^{\xi, x}(y)\right) d y,
$$

where $v^{\xi, x}$ is the solution of the cell-problem

$$
\left\{\begin{array}{l}
-\operatorname{div}\left(a\left(x, y, \xi+D v^{\xi, x}(y)\right)\right)=0 \quad \text { on } \quad Y, \\
v^{\xi, x} \in H_{\square}^{1,2}(Y),
\end{array}\right.
$$

where $Y$ is a cell of periodicity and $H_{\square}^{1,2}(Y)$ is the subset of $H^{1,2}(Y)$ such that $u$ has mean value 0 and $u$ is $Y$-periodic. The homogenization problem for monotone operators of this type has been studied by several authors but with no dependence in $x$, i.e. $a$ is on the form $a(x, y, \xi)=a(y, \xi)$. Here we mention [7] where the problem was studied in the Sobolev space $H^{1, p}, 1<p<\infty$, with appropriate continuity and monotonicity conditions. In [⿶] the corresponding multi-valued case was considered. Moreover, the almost periodic case was treated in [3].

The weak convergence of $u_{h}$ to $u$ in $H^{1,2}(\Omega)$ implies that $u_{h}-u \rightarrow 0$ in $L^{2}(\Omega)$ but in general we only have that $D u_{h}-D u \rightarrow 0$ weakly in $L^{2}\left(\Omega, R^{n}\right)$. However, we will prove that it is possible to express $D u_{h}$ in terms of $D u$, up to a rest which converges strongly in $L^{2}\left(\Omega, R^{n}\right)$. This is done by constructing a family of correctors $P_{h}(x, \xi, t)$, defined by

$$
P_{h}(x, \xi, t)=P\left(\frac{x}{\varepsilon_{h}}, \xi, t\right)=\xi+D v^{\xi, t}\left(\frac{x}{\varepsilon_{h}}\right) .
$$

Let $\left(M_{h}\right)$ be a family of linear operators converging to the identity map on $L^{2}\left(\Omega, R^{n}\right)$ such that $M_{h} f$ is a step function for every $f \in L^{2}\left(\Omega, R^{n}\right)$. Moreover, let $\gamma_{h}$ be a step function approximating the identity map on $\Omega$. We will show that

$$
D u_{h}-P_{h}\left(x, M_{h} D u, \gamma_{h}\right) \rightarrow 0 \quad \text { in } \quad L^{2}\left(\Omega, R^{n}\right) .
$$

Results concerning correctors for an even more general case than studied in [7] can be found in [5]. The almost periodic case has been studied in [2]. 


\section{Preliminaries and notations}

Let $\Omega$ be a open bounded subset of $R^{n},|E|$ denotes the Lebesgue measure in $R^{n}$ and $(\cdot, \cdot)$ is the Euclidean scalar product on $R^{n}$. Let $\left\{\Omega_{i} \subset \Omega: i=1, \ldots, N\right\}$ be a family of disjoint open sets such that $\left|\Omega \backslash \cup_{i=1}^{N} \Omega_{i}\right|=0$ and $\left|\partial \Omega_{i}\right|=0$. Let $\left(\varepsilon_{h}\right)$ be a decreasing sequence of real numbers such that $\varepsilon_{h} \rightarrow 0$ as $h \rightarrow \infty$. $Y=(0,1)^{n}$ is the unit cube in $R^{n}$ and $Y_{h}^{j}=\varepsilon_{h}(j+Y)$, where $j \in Z^{n}$, i.e. the translated image of $\varepsilon_{h} Y$ by the vector $\varepsilon_{h} j$. We also define the following index sets:

$$
\begin{aligned}
& J_{h}=\left\{j \in Z^{n}: \bar{Y}_{h}^{j} \subset \Omega\right\}, J_{h}^{i}=\left\{j \in Z^{n}: \bar{Y}_{h}^{j} \subset \Omega_{i}\right\}, \\
& B_{h}^{i}=\left\{j \in Z^{n}: \bar{Y}_{h}^{j} \cap \Omega_{i} \neq \emptyset, \bar{Y}_{h}^{j} \backslash \Omega_{i} \neq \emptyset\right\} .
\end{aligned}
$$

Moreover, we define $\Omega_{i}^{h}=\cup_{j \in J_{h}^{i}} \bar{Y}_{h}^{j}$ and $F_{i}^{h}=\cup_{j \in B_{h}^{i}} Y_{h}^{j}$.

In a corresponding way let $\left\{\Omega_{i}^{k} \subset \Omega: i \in I_{k}\right\}$ denote a family of disjoint open sets with diameter less than $\frac{1}{k}$ such that $\left|\Omega \backslash \cup_{i \in I_{k}} \Omega_{i}^{k}\right|=0$ and $\left|\partial \Omega_{i}^{k}\right|=0$. We also define the following index sets:

$$
\begin{aligned}
& J_{h}^{i, k}=\left\{j \in Z^{n}: \bar{Y}_{h}^{j} \subset \Omega_{i}^{k}\right\}, \\
& B_{h}^{i, k}=\left\{j \in Z^{n}: \bar{Y}_{h}^{j} \cap \Omega_{i}^{k} \neq \emptyset, \bar{Y}_{h}^{j} \backslash \Omega_{i}^{k} \neq \emptyset\right\} .
\end{aligned}
$$

Let $\Omega_{i}^{k, h}=\cup_{j \in J_{h}^{i, k}} \bar{Y}_{h}^{j}$ and $F_{i}^{k, h}=\cup_{j \in B_{h}^{i, k}} Y_{h}^{j}$.

Corresponding to $f \in L^{2}\left(\Omega, R^{n}\right)$ we define the function $M_{h} f: R^{n} \rightarrow R^{n}$ by

$$
\left(M_{h} f\right)(x)=\sum_{j \in J_{h}} \chi_{Y_{h}^{j}}(x) \xi_{h}^{j}
$$

where $\xi_{h}^{j}=\frac{1}{\left|Y_{h}^{j}\right|} \int_{Y_{h}^{j}} f d x$ and $\chi_{E}$ is the characteristic function of the set $E$ (in order to define $\xi_{h}^{j}$ for all $j \in Z^{n}$ we treat $f$ as $f=0$ outside $\Omega$ ). It is well known that

$$
M_{h} f \rightarrow f \quad \text { in } \quad L^{2}\left(\Omega, R^{n}\right),
$$

see [11], page 129. We also define the step function $\gamma_{h}: \Omega \rightarrow \Omega$ by

$$
\gamma_{h}(x)=\sum_{j \in J_{h}} \chi_{Y_{h}^{j}}(x) x_{h}^{j}
$$

where $x_{h}^{j} \in Y_{h}^{j}$. Moreover, $C$ will be a constant that may differ from one place to an other. Let $a: \Omega \times R^{n} \times R^{n} \rightarrow R^{n}$ be a function such that $a(x, \cdot, \xi)$ is Lebesgue measurable and $Y$ periodic for $x \in \Omega$ and $\xi \in R^{n}$. We also assume that $a$ satisfies the following monotonicity and continuity conditions: There exists two constants $0<\alpha \leq \beta<\infty$ such that

$$
\begin{aligned}
& \left(a\left(x, y, \xi_{1}\right)-a\left(x, y, \xi_{2}\right), \xi_{1}-\xi_{2}\right) \geq \alpha\left|\xi_{1}-\xi_{2}\right|^{2}, \\
& \mid a\left(x, y, \xi_{1}-a\left(x, y, \xi_{2}\right)|\leq \beta| \xi_{1}-\xi_{2} \mid\right.
\end{aligned}
$$


for $x \in \Omega$, a.e. $y \in R^{n}$ and every $\xi \in R^{n}$. Moreover we assume that

$$
a(x, y, 0)=0,
$$

for $x \in \Omega$, a.e. $y \in R^{n}$. Let $\left(f_{h}\right)$ be a sequence in $H^{-1,2}(\Omega)$ which converges to $f$.

The solution $v^{\xi, x}$ of the cell-problem (11) can be extended by periodicity to an element in $H_{\text {loc }}^{1,2}\left(R^{n}\right)$, still denoted by $v^{\xi, x}$ and

$$
\int_{R^{n}}\left(a\left(x, y, \xi+D v^{\xi, x}(y)\right), D \phi(y)\right) d y=0 \quad \text { for every } \quad \phi \in C_{0}^{\infty}\left(R^{n}\right) .
$$

The following compensated compactness lemma will be used frequently, see [⿰], page 4 .

Lemma 1. Let $1<p<\infty$. Moreover, let $\left(v_{h}\right)$ be a sequence in $L^{q}\left(\Omega, R^{n}\right)$ which converges weakly to $v,\left(-\operatorname{div} v_{h}\right)$ converges to $-\operatorname{div} v$ in $W^{-1, q}(\Omega)$ and let $\left(u_{h}\right)$ be a sequence which converges weakly to $u$ in $W^{1, p}(\Omega)$. Then

$$
\int_{\Omega}\left(v_{h}, D u_{h}\right) \phi d x \rightarrow \int_{\Omega}(v, D u) \phi d x
$$

for every $\phi \in C_{0}^{\infty}(\Omega)$.

\section{Some homogenization results}

Let $a(x, y, \xi)$ satisfy (5), (6), (7) and one of the following conditions:

(i) $a$ is on the form

$$
a(x, y, \xi)=\sum_{i=1}^{N} \chi_{\Omega_{i}}(x) a_{i}(y, \xi)
$$

(ii) there exist a function $\omega: R \rightarrow R$ which is continuous, increasing and $\omega(0)=0$ such that

$$
\mid a\left(x_{1}, y, \xi-\left.a\left(x_{2}, y, \xi\right)\right|^{2} \leq \omega\left(\left|x_{1}-x_{2}\right|\right)|\xi|^{2}\right.
$$

for $x \in \Omega$, a.e. $y \in R^{n}$ and every $\xi \in R^{n}$.

Now we consider the weak Dirichlet boundary value problems, one for each $h$,

$$
\left\{\begin{array}{l}
\int_{\Omega}\left(a\left(x, \frac{x}{\varepsilon_{h}}, D u_{h}\right), D \phi\right) d x=\left\langle f_{h}, \phi\right\rangle \quad \text { for every } \phi \in H_{0}^{1,2}(\Omega), \\
u_{h} \in H_{0}^{1,2}(\Omega) .
\end{array}\right.
$$

By a standard result in the existence theory for boundary value problems defined by monotone operators these problems have unique solution for each $h$, see e.g. [14]. Furthermore, 
by choosing $\phi=u_{h}$ in (11), taking into account (5), (7) and using the fact that $\left(f_{h}\right)$ is bounded we have that

$$
\begin{aligned}
\alpha \int_{\Omega}\left|D u_{h}\right|^{2} d x & \leq \int_{\Omega}\left(a\left(x, \frac{x}{\varepsilon_{h}}, D u_{h}\right), D u_{h}\right) d x=\left\langle f_{h}, u_{h}\right\rangle \\
& \leq\left\|f_{h}\right\|_{H^{-1,2}(\Omega)}\left\|u_{h}\right\|_{H_{0}^{1,2}(\Omega)} \leq C\left\|u_{h}\right\|_{H_{0}^{1,2}(\Omega)},
\end{aligned}
$$

where $C$ does not depend on $h$. The Poincaré inequality then implies that

$$
\left\|u_{h}\right\|_{H_{0}^{1,2}(\Omega)} \leq C
$$

where $C$ does not depend on $h$. Therefore there exists a subsequence $\left(h^{\prime}\right)$ such that

$$
u_{h^{\prime}} \rightarrow u_{*} \quad \text { weakly in } H_{0}^{1,2}(\Omega) .
$$

It is now natural to raise the following question: does $u_{*}$ satisfy an equation of the same type as that satisfied by $u_{h}$ ? The answer to this question is given in the following theorems:

Theorem 1. Let a satisfy (5), (6), (19) and (9). Moreover, let $\left(u_{h}\right)$ be the solutions of (11). Then

$$
\begin{aligned}
& u_{h} \rightarrow u \quad \text { weakly in } H_{0}^{1,2}(\Omega), \\
& a\left(x, \frac{x}{\varepsilon_{h}}, D u_{h}\right) \rightarrow b(x, D u) \quad \text { weakly in } L^{2}\left(\Omega ; R^{n}\right),
\end{aligned}
$$

where $u$ is the unique solution of the homogenized problem

$$
\left\{\begin{array}{l}
\int_{\Omega}(b(x, D u), D \phi) d x=\langle f, \phi\rangle \quad \text { for every } \quad \phi \in H_{0}^{1,2}(\Omega), \\
u \in H_{0}^{1,2}(\Omega) .
\end{array}\right.
$$

The operator $b: \Omega \times R^{n} \rightarrow R^{n}$ is defined a.e. as

$$
b(x, \xi)=\sum_{i=1}^{N} \chi_{\Omega_{i}}(x) \int_{Y} a_{i}\left(y, \xi+D v^{\xi, x_{i}}(y)\right) d y=\sum_{i=1}^{N} \chi_{\Omega_{i}}(x) b_{i}(\xi),
$$

where $x_{i} \in \Omega_{i}$ and $v^{\xi, x_{i}}$ is the unique solution of the cell problem

$$
\left\{\begin{array}{l}
\int_{Y}\left(a_{i}\left(y, \xi+D v^{\xi, x_{i}}(y)\right), D \phi(y)\right) d y=0 \quad \text { for every } \quad \phi \in H_{\square}^{1,2}(Y), \\
v^{\xi, x_{i}} \in H_{\square}^{1,2}(Y) .
\end{array}\right.
$$

Proof. The proof follows by using the ideas in [6] where the case $N=1$ is treated for the details the reader is referred to [12].

Theorem 2. Let a satisfy (鸟), (6), (1) and (10). Moreover, let $\left(u_{h}\right)$ be the solutions of (11). Then

$$
u_{h} \rightarrow u \quad \text { weakly in } H_{0}^{1,2}(\Omega),
$$




$$
a\left(x, \frac{x}{\varepsilon_{h}}, D u_{h}\right) \rightarrow b(x, D u) \quad \text { weakly in } \quad L^{2}\left(\Omega ; R^{n}\right),
$$

where $u$ is the unique solution of

$$
\left\{\begin{array}{l}
\int_{\Omega}(b(x, D u), D \phi) d x=\left\langle f_{h}, \phi\right\rangle \quad \text { for every } \phi \in H_{0}^{1,2}(\Omega), \\
u \in H_{0}^{1,2}(\Omega) .
\end{array}\right.
$$

The operator $b: \Omega \times R^{n} \rightarrow R^{n}$ is defined as

$$
b(x, \xi)=\int_{Y} a\left(x, y, \xi+D v^{\xi, x}(y)\right) d y,
$$

where $v^{\xi, x}$ is the unique solution of the cell-problem

$$
\left\{\begin{array}{l}
\int_{Y}\left(a\left(x, y, \xi+D v^{\xi, x}(y)\right), D \phi\right) d y=0 \quad \text { for every } \phi \in H_{\square}^{1,2}(Y), \\
v^{\xi, x} \in H_{\square}^{1,2}(Y) .
\end{array}\right.
$$

Before we prove this theorem we make some definitions and prove some lemmas that will be useful in the proof. Define the function

$$
a^{k}(x, y, \xi):=\sum_{i \in I_{k}} \chi_{\Omega_{i}^{k}}(x) a\left(x_{i}^{k}, y, \xi\right),
$$

where $x_{i}^{k} \in \Omega_{i}^{k}$. Consider the boundary value problems

$$
\left\{\begin{array}{l}
\int_{\Omega}\left(a^{k}\left(x, \frac{x}{\varepsilon_{h}}, D u_{h}^{k}\right), D \phi\right) d x=\left\langle f_{h}, \phi\right\rangle \quad \text { for every } \phi \in H_{0}^{1,2}(\Omega), \\
u_{h}^{k} \in H_{0}^{1,2}(\Omega) .
\end{array}\right.
$$

The conditions for Theorem 1 are satisfied and the theorem implies that there exists a $u_{*}^{k}$ such that

$$
u_{h}^{k} \rightarrow u_{*}^{k} \quad \text { weakly in } H_{0}^{1,2}(\Omega) \text { as } h \rightarrow \infty,
$$

and $u_{*}^{k}$ is the unique solution of

$$
\left\{\begin{array}{l}
\int_{\Omega}\left(b^{k}\left(x, D u_{*}^{k}\right), D \phi\right) d x=\langle f, \phi\rangle \quad \text { for every } \phi \in H_{0}^{1,2}(\Omega), \\
u_{*}^{k} \in H_{0}^{1,2}(\Omega)
\end{array}\right.
$$

where

$$
b^{k}(x, \xi)=\sum_{i \in I_{k}} \chi_{\Omega_{i}^{k}}(x) \int_{Y} a\left(x_{i}^{k}, y, \xi+D v^{\xi, x_{i}^{k}}(y)\right) d y=\sum_{i \in I_{k}} \chi_{\Omega_{i}^{k}}(x) b\left(x_{i}^{k}, \xi\right),
$$

where $v^{\xi, x_{i}^{k}}$ is the solution of

$$
\left\{\begin{array}{l}
\int_{Y}\left(a\left(x_{i}^{k}, y, \xi+D v^{\xi, x_{i}^{k}}(y)\right), D \phi\right) d y=0 \quad \text { for every } \quad \phi \in H_{\square}^{1,2}(Y), \\
v^{\xi, x_{i}^{k}} \in H_{\square}^{1,2}(Y) .
\end{array}\right.
$$


Proof of Theorem 2. First we prove that $u_{h} \rightarrow u$ weakly in $H_{0}^{1,2}(\Omega)$. If $g \in H^{-1,2}(\Omega)$, then

$$
\begin{aligned}
& \lim _{h \rightarrow \infty}\left\langle g, u_{h}-u\right\rangle=\lim _{k \rightarrow \infty} \lim _{h \rightarrow \infty}\left\langle g, u_{h}-u\right\rangle=\lim _{k \rightarrow \infty} \lim _{h \rightarrow \infty}\left\langle g, u_{h}-u_{h}^{k}+u_{h}^{k}-u_{*}^{k}+u_{*}^{k}-u\right\rangle \\
& \quad \leq \lim _{k \rightarrow \infty} \lim _{h \rightarrow \infty}\|g\|_{H^{-1,2}(\Omega)}\left\|u_{h}-u_{h}^{k}\right\|_{H_{0}^{1,2}(\Omega)}+\lim _{k \rightarrow \infty} \lim _{h \rightarrow \infty}\left\langle g, u_{h}^{k}-u_{*}^{k}\right\rangle \\
& \quad+\lim _{k \rightarrow \infty}\|g\|_{H^{-1,2}(\Omega)}\left\|u_{*}^{k}-u\right\|_{H_{0}^{1,2}(\Omega)} .
\end{aligned}
$$

It is enough to prove that all three terms on the right hand side are zero. We do this in three steps.

Step 1. Let us prove that

$$
\lim _{k \rightarrow \infty} \lim _{h \rightarrow \infty}\left\|u_{h}-u_{h}^{k}\right\|_{H_{0}^{1,2}(\Omega)}=0
$$

By definition

$$
\begin{aligned}
& \int_{\Omega}\left(a^{k}\left(x, \frac{x}{\varepsilon_{h}}, D u_{h}^{k}\right), D \phi\right) d x=\left\langle f_{h}, \phi\right\rangle \quad \text { for every } \phi \in H_{0}^{1,2}(\Omega), \\
& \int_{\Omega}\left(a\left(x, \frac{x}{\varepsilon_{h}}, D u_{h}\right), D \phi\right) d x=\left\langle f_{h}, \phi\right\rangle \quad \text { for every } \quad \phi \in H_{0}^{1,2}(\Omega) .
\end{aligned}
$$

This implies that we for $\phi=u_{h}^{k}-u_{h}$ have

$$
\begin{aligned}
\int_{\Omega} & \left(a^{k}\left(x, \frac{x}{\varepsilon_{h}}, D u_{h}^{k}\right)-a^{k}\left(x, \frac{x}{\varepsilon_{h}}, D u_{h}\right), D u_{h}^{k}-D u_{h}\right) d x \\
= & \int_{\Omega}\left(a\left(x, \frac{x}{\varepsilon_{h}}, D u_{h}\right)-a^{k}\left(x, \frac{x}{\varepsilon_{h}}, D u_{h}\right), D u_{h}^{k}-D u_{h}\right) d x
\end{aligned}
$$

According to the monotonicity of $a$, (5), the Schwarz inequality and the Hölder inequality we obtain that

$$
\begin{aligned}
& \alpha \int_{\Omega}\left|D u_{h}^{k}-D u_{h}\right|^{2} d x \\
& \quad \leq\left(\int_{\Omega}\left|a\left(x, \frac{x}{\varepsilon_{h}}, D u_{h}\right)-a^{k}\left(x, \frac{x}{\varepsilon_{h}}, D u_{h}\right)\right|^{2} d x\right)^{\frac{1}{2}}\left(\int_{\Omega}\left|D u_{h}^{k}-D u_{h}\right|^{2} d x\right)^{\frac{1}{2}}
\end{aligned}
$$

i.e.

$$
\left\|D u_{h}^{k}-D u_{h}\right\|_{L^{2}\left(\Omega ; R^{n}\right)}^{2} \leq \frac{1}{\alpha^{2}} \int_{\Omega}\left|a\left(x, \frac{x}{\varepsilon_{h}}, D u_{h}\right)-a^{k}\left(x, \frac{x}{\varepsilon_{h}}, D u_{h}\right)\right|^{2} d x .
$$

Thus, in view of the continuity condition (10) on $a$, we get

$$
\left\|D u_{h}^{k}-D u_{h}\right\|_{L^{2}\left(\Omega ; R^{n}\right)}^{2} \leq \frac{1}{\alpha^{2}} \omega\left(\frac{1}{k}\right) \int_{\Omega}\left|D u_{h}\right|^{2} d x \leq \frac{C}{\alpha^{2}} \omega\left(\frac{1}{k}\right),
$$


where we in the last inequality used the fact that there exists a constant $C$ independent of $h$ such that $\left\|D u_{h}\right\|_{L^{2}\left(\Omega ; R^{n}\right)}^{2} \leq C$. Since $\|D \cdot\|_{L^{2}\left(\Omega ; R^{n}\right)}^{2}$ is an equivalent norm on $H_{0}^{1,2}(\Omega)$ (21) implies that

$$
\left\|u_{h}^{k}-u_{h}\right\|_{H_{0}^{1,2}(\Omega)} \rightarrow 0
$$

as $k \rightarrow \infty$ uniformly in $h$. This means that we can change the order in the limit process in (20) and (20) follows by taking (21) into account.

Step 2. We observe that

$$
\lim _{k \rightarrow \infty} \lim _{h \rightarrow \infty}\left\langle g, u_{h}^{k}-u_{*}^{k}\right\rangle=0
$$

as a direct consequence of Theorem 1 .

Step 3. Let us prove that

$$
\lim _{k \rightarrow \infty}\left\|u_{*}^{k}-u\right\|_{H_{0}^{1,2}(\Omega)}=0
$$

By definition we have that

$$
\begin{aligned}
& \int_{\Omega}\left(b^{k}\left(x, D u_{*}^{k}\right), D \phi\right) d x=\langle f, \phi\rangle \quad \text { for every } \phi \in H_{0}^{1,2}(\Omega), \\
& \int_{\Omega}(b(x, D u), D \phi) d x=\langle f, \phi\rangle \quad \text { for every } \phi \in H_{0}^{1,2}(\Omega) .
\end{aligned}
$$

Thus

$$
\int_{\Omega}\left(b^{k}\left(x, D u_{*}^{k}\right)-b^{k}(x, D u), D \phi\right) d x=\int_{\Omega}\left(b(x, D u)-b^{k}(x, D u), D \phi\right) d x,
$$

for every $\phi \in H_{0}^{1,2}(\Omega)$. Choose $\phi=u_{*}^{k}-u$ and take the strict monotonicity of $b^{k}$, see Remark 1 in the next section, into account on the left hand side and apply the Schwarz inequality and Hölder inequality on the right hand side to obtain

$$
\left.\alpha \int_{\Omega}\left|D u_{*}^{k}-D u\right|^{2} d x \leq\left.\left(\int_{\Omega}\left|b(x, D u)-b^{k}(x, D u)\right|^{2} d x\right)^{\frac{1}{2}}\left(\int_{\Omega} \mid D u_{*}^{k}-D u\right)\right|^{2} d x\right)^{\frac{1}{2}} .
$$

Hence, by Theorem 3

$$
\left.\left.\left(\int_{\Omega} \mid D u_{*}^{k}-D u\right)\right|^{2} d x\right)^{\frac{1}{2}} \leq \frac{1}{\alpha}\left(\omega\left(\frac{1}{k}\right) C \int_{\Omega}|D u|^{2} d x\right)^{\frac{1}{2}} .
$$

The right hand side tends to 0 as $k \rightarrow \infty$. We obtain (3) by noting that $\|D \cdot\|_{L^{2}\left(\Omega ; R^{n}\right)}^{2}$ is an equivalent norm on $H_{0}^{1,2}(\Omega)$. 
Next we prove that $a\left(x, \frac{x}{\varepsilon_{h}}, D u_{h}\right) \rightarrow b(x, D u)$ weakly in $L^{2}\left(\Omega ; R^{n}\right)$. In fact if $g \in$ $\left(L^{2}\left(\Omega ; R^{n}\right)\right)^{*}$, then

$$
\begin{aligned}
& \lim _{h \rightarrow \infty}\left\langle g, a\left(x, \frac{x}{\varepsilon_{h}}, D u_{h}\right)-b(x, D u)\right\rangle=\lim _{k \rightarrow \infty} \lim _{h \rightarrow \infty}\left\langle g, a\left(x, \frac{x}{\varepsilon_{h}}, D u_{h}\right)-b(x, D u)\right\rangle \\
& \quad=\lim _{k \rightarrow \infty} \lim _{h \rightarrow \infty}\left\langle g, a\left(x, \frac{x}{\varepsilon_{h}}, D u_{h}\right)-a^{k}\left(x, \frac{x}{\varepsilon_{h}}, D u_{h}^{k}\right)\right\rangle \\
& \quad+\lim _{k \rightarrow \infty} \lim _{h \rightarrow \infty}\left\langle g, a^{k}\left(x, \frac{x}{\varepsilon_{h}}, D u_{h}^{k}\right)-b^{k}\left(x, D u_{*}^{k}\right)\right\rangle \\
& \quad+\lim _{k \rightarrow \infty} \lim _{h \rightarrow \infty}\left\langle g, b^{k}\left(x, D u_{*}^{k}\right)-b(x, D u)\right\rangle \\
& \leq \lim _{k \rightarrow \infty} \lim _{h \rightarrow \infty}\|g\|_{\left(L^{2}\left(\Omega ; R^{n}\right)\right)^{*}}\left\|a\left(x, \frac{x}{\varepsilon_{h}}, D u_{h}\right)-a^{k}\left(x, \frac{x}{\varepsilon_{h}}, D u_{h}^{k}\right)\right\|_{L^{2}\left(\Omega ; R^{n}\right)} \\
& \left.\quad+\lim _{k \rightarrow \infty} \lim _{h \rightarrow \infty}\left\langle g, a^{k}\left(x, \frac{x}{\varepsilon_{h}}, D u_{h}^{k}\right)-b^{k}\left(x, D u_{*}^{k}\right)\right)\right\rangle \\
& \quad+\lim _{k \rightarrow \infty}\|g\|_{\left(L^{2}\left(\Omega ; R^{n}\right)\right)^{*}}\left\|b^{k}\left(x, D u_{*}^{k}\right)-b(x, D u)\right\|_{L^{2}\left(\Omega ; R^{n}\right)}
\end{aligned}
$$

It is sufficient to prove that all three terms on the right hand side are zero. We do this in three steps.

Step 1. Let us show that

$$
\lim _{k \rightarrow \infty} \lim _{h \rightarrow \infty}\left\|a\left(x, \frac{x}{\varepsilon_{h}}, D u_{h}\right)-a^{k}\left(x, \frac{x}{\varepsilon_{h}}, D u_{h}^{k}\right)\right\|_{L^{2}\left(\Omega ; R^{n}\right)}=0 .
$$

By using elementary estimates we find that

$$
\begin{aligned}
& \int_{\Omega}\left|a^{k}\left(x, \frac{x}{\varepsilon_{h}}, D u_{h}^{k}\right)-a\left(x, \frac{x}{\varepsilon_{h}}, D u_{h}\right)\right|^{2} d x \\
& =\int_{\Omega}\left|a^{k}\left(x, \frac{x}{\varepsilon_{h}}, D u_{h}^{k}\right)-a^{k}\left(x, \frac{x}{\varepsilon_{h}}, D u_{h}\right)+a^{k}\left(x, \frac{x}{\varepsilon_{h}}, D u_{h}\right)-a\left(x, \frac{x}{\varepsilon_{h}}, D u_{h}\right)\right|^{2} d x \\
& \leq 2 \int_{\Omega}\left|a^{k}\left(x, \frac{x}{\varepsilon_{h}}, D u_{h}^{k}\right)-a^{k}\left(x, \frac{x}{\varepsilon_{h}}, D u_{h}\right)\right|^{2} d x \\
& +2 \int_{\Omega}\left|a^{k}\left(x, \frac{x}{\varepsilon_{h}}, D u_{h}\right)-a\left(x, \frac{x}{\varepsilon_{h}}, D u_{h}\right)\right|^{2} d x .
\end{aligned}
$$

Hence, by applying the continuity conditions (6) and (10), we obtain that

$$
\begin{aligned}
\int_{\Omega} \mid & a^{k}\left(x, \frac{x}{\varepsilon_{h}}, D u_{h}^{k}\right)-\left.a\left(x, \frac{x}{\varepsilon_{h}}, D u_{h}\right)\right|^{2} d x \\
& \leq 2 \beta^{2} \int_{\Omega}\left|D u_{h}^{k}-D u_{h}\right|^{2} d x+2 \omega\left(\frac{1}{k}\right) \int_{\Omega}\left|D u_{h}\right|^{2} d x .
\end{aligned}
$$


According to (21) and the fact that $\left(D u_{h}\right)$ is bounded in $L^{2}\left(\Omega ; R^{n}\right)$ there exists a constant $C$ independent of $h$ such that

$$
\int_{\Omega}\left|a^{k}\left(x, \frac{x}{\varepsilon_{h}}, D u_{h}^{k}\right)-a\left(x, \frac{x}{\varepsilon_{h}}, D u_{h}\right)\right|^{2} d x \leq C \omega\left(\frac{1}{k}\right) .
$$

By the properties of $\omega$ it follows that

$$
\left\|a\left(x, \frac{x}{\varepsilon_{h}}, D u_{h}\right)-a^{k}\left(x, \frac{x}{\varepsilon_{h}}, D u_{h}^{k}\right)\right\|_{L^{2}\left(\Omega ; R^{n}\right)} \rightarrow 0,
$$

as $k \rightarrow \infty$ uniformly in $h$. This implies that we may change the order in the limit process in (24) and we obtain (24) by taking (25) into account.

Step 2. We observe that

$$
\lim _{k \rightarrow \infty} \lim _{h \rightarrow \infty}\left\langle g, a^{k}\left(x, \frac{x}{\varepsilon_{h}}, D u_{h}^{k}\right)-b^{k}\left(x, D u_{*}^{k}\right)\right\rangle=0,
$$

as a direct consequence of Theorem 1 .

Step 3. Let us show that

$$
\lim _{k \rightarrow \infty}\left\|b^{k}\left(x, D u_{*}^{k}\right)-b(x, D u)\right\|_{L^{2}\left(\Omega ; R^{n}\right)}=0 .
$$

We have that

$$
\begin{aligned}
\int_{\Omega}\left|b^{k}\left(x, D u_{*}^{k}\right)-b(x, D u)\right|^{2} d x \\
\quad=\int_{\Omega}\left|b^{k}\left(x, D u_{*}^{k}\right)-b^{k}(x, D u)+b^{k}(x, D u)-b(x, D u)\right|^{2} d x \\
\quad \leq 2 \int_{\Omega}\left|b^{k}\left(x, D u_{*}^{k}\right)-b^{k}(x, D u)\right|^{2} d x+2 \int_{\Omega}\left|b^{k}(x, D u)-b(x, D u)\right|^{2} d x .
\end{aligned}
$$

By applying the continuity conditions in Remark 1 and Theorem 3 we see that

$$
\int_{\Omega}\left|b^{k}\left(x, D u_{*}^{k}\right)-b(x, D u)\right|^{2} d x \leq 2 \frac{\beta^{4}}{\alpha^{2}} \int_{\Omega}\left|D u_{*}^{k}-D u\right|^{2} d x+2 C \omega\left(\frac{1}{k}\right) \int_{\Omega}|D u|^{2} d x .
$$

Now (26) follows by taking (23) into account.

\section{Properties of the homogenized operator}

In this section we prove some properties of the homogenized operator. In particular these properties implies the existence and uniqueness of the solution of the homogenized problem.

Theorem 3. Let b be the homogenized operator defined in Theorem 2. Then

(a) $b(\cdot, \xi)$ satisfies the continuity condition

$$
\left|b\left(x_{1}, \xi\right)-b\left(x_{2}, \xi\right)\right|^{2} \leq \omega\left(\left|x_{1}-x_{2}\right|\right) C|\xi|^{2},
$$

where $C=2\left(\frac{\beta}{\alpha}\right)^{2}\left(1+\left(\frac{\beta}{\alpha}\right)^{2}\right)$. 
(b) $b(x, \cdot)$ is strictly monotone, more precisely

$$
\left(b\left(x, \xi_{1}\right)-b\left(x, \xi_{2}\right), \xi_{1}-\xi_{2}\right) \geq \alpha\left|\xi_{1}-\xi_{2}\right|^{2}, \quad \text { for every } \xi_{1}, \xi_{2} \in R^{n}
$$

(c) $b(x, \cdot)$ is Lipschitz continuous, more precisely

$$
\left|b\left(x, \xi_{1}\right)-b\left(x, \xi_{2}\right)\right| \leq \frac{\beta^{2}}{\alpha}\left|\xi_{1}-\xi_{2}\right|, \quad \text { for every } \quad \xi_{1}, \xi_{2} \in R^{n}
$$

(d) $b(x, 0)=0$ for $x \in \Omega$.

Proof. (a) By the definition of $b$ we have

$$
\begin{aligned}
& \left|b\left(x_{1}, \xi\right)-b\left(x_{2}, \xi\right)\right|^{2}=\left|\int_{Y} a\left(x_{1}, y, \xi+D v^{\xi, x_{1}}\right) d y-\int_{Y} a\left(x_{2}, y, \xi+D v^{\xi, x_{2}}\right) d y\right|^{2} \\
& \quad \leq\left(\int_{Y}\left|a\left(x_{1}, y, \xi+D v^{\xi, x_{1}}\right)-a\left(x_{2}, y, \xi+D v^{\xi, x_{1}}\right)\right| d y\right. \\
& \left.\quad+\int_{Y}\left|a\left(x_{2}, y, \xi+D v^{\xi, x_{1}}\right)-a\left(x_{2}, y, \xi+D v^{\xi, x_{2}}\right)\right| d y\right)^{2} \\
& \quad \leq 2\left(\int_{Y}\left|a\left(x_{1}, y, \xi+D v^{\xi, x_{1}}\right)-a\left(x_{2}, y, \xi+D v^{\xi, x_{1}}\right)\right| d y\right) \\
& \quad+2\left(\int_{Y}\left|a\left(x_{2}, y, \xi+D v^{\xi, x_{1}}\right)-a\left(x_{2}, y, \xi+D v^{\xi, x_{2}}\right)\right| d y\right)^{2}
\end{aligned}
$$

where we in the last inequality used that $(a+b)^{2} \leq 2\left(a^{2}+b^{2}\right)$ for $a, b \geq 0$. By the Jensen inequality we get

$$
\begin{aligned}
& \left|b\left(x_{1}, \xi\right)-b\left(x_{2}, \xi\right)\right|^{2} \leq 2 \int_{Y}\left|a\left(x_{1}, y, \xi+D v^{\xi, x_{1}}\right)-a\left(x_{2}, y, \xi+D v^{\xi, x_{1}}\right)\right|^{2} d y \\
& \quad+2 \int_{Y}\left|a\left(x_{2}, y, \xi+D v^{\xi, x_{1}}\right)-a\left(x_{2}, y, \xi+D v^{\xi, x_{2}}\right)\right|^{2} d y .
\end{aligned}
$$

From the continuity conditions (10) and (6) it follows that

$$
\begin{aligned}
& \left|b\left(x_{1}, \xi\right)-b\left(x_{2}, \xi\right)\right|^{2} \\
& \quad \leq 2 \omega\left(\left|x_{1}-x_{2}\right|\right) \int_{Y}\left|\xi+D v^{\xi, x_{1}}\right|^{2} d y+2 \beta^{2} \int_{Y}\left|D v^{\xi, x_{1}}-D v^{\xi, x_{2}}\right|^{2} d y .
\end{aligned}
$$

We will now study the two terms in (27) separately. 
First term: According to (5), (16), (6) we have

$$
\begin{aligned}
& \alpha \int_{Y}\left|\xi_{1}+D v^{\xi_{1}, x}(y)-\xi_{2}-D v^{\xi_{2}, x}(y)\right|^{2} d y \\
& \leq \int_{Y}\left(a\left(x, y, \xi_{1}+D v^{\xi_{1}, x}\right)-a\left(x, y, \xi_{2}+D v^{\xi_{2}, x}\right), \xi_{1}+D v^{\xi_{1}, x}-\xi_{2}-D v^{\xi_{2}, x}\right) d y \\
& =\int_{Y}\left(a\left(x, y, \xi_{1}+D v^{\xi_{1}, x}\right)-a\left(x, y, \xi_{2}+D v^{\xi_{2}, x}\right), \xi_{1}-\xi_{2}\right) d y \\
& \leq \int_{Y}\left|a\left(x, y, \xi_{1}+D v^{\xi_{1}, x}\right)-a\left(x, y, \xi_{2}+D v^{\xi_{2}, x}\right)\right|\left|\xi_{1}-\xi_{2}\right| d y \\
& \left.\leq \beta \int_{Y} \mid \xi_{1}+D v^{\xi_{1}, x}-\xi_{2}-D v^{\xi_{2}, x}\right)|| \xi_{1}-\xi_{2} \mid d y \\
& \left.\leq\left.\beta\left(\int_{Y} \mid \xi_{1}+D v^{\xi_{1}, t}-\xi_{2}-D v^{\xi_{2}, t}\right)\right|^{2} d y\right)^{\frac{1}{2}}\left(\int_{Y}\left|\xi_{1}-\xi_{2}\right|^{2} d y\right)^{\frac{1}{2}} .
\end{aligned}
$$

This can be written as

$$
\int_{Y}\left|\xi_{1}+D v^{\xi_{1}, t}(y)-\xi_{2}-D v^{\xi_{2}, t}(y)\right|^{2} d y \leq\left(\frac{\beta}{\alpha}\right)^{2}\left|\xi_{1}-\xi_{2}\right|^{2} .
$$

Second term: By definition we have that

$$
\begin{aligned}
& \int_{Y}\left(a\left(x_{1}, y, \xi+D v^{\xi, x_{1}}(y)\right), D \phi\right) d y=0 \text { for every } \phi \in H_{\square}^{1,2}(\Omega), \\
& \int_{Y}\left(a\left(x_{2}, y, \xi+D v^{\xi, x_{2}}(y)\right), D \phi\right) d y=0 \text { for every } \phi \in H_{\square}^{1,2}(\Omega) .
\end{aligned}
$$

This implies that

$$
\begin{aligned}
\int_{Y} & \left(a\left(x_{1}, y, \xi+D v^{\xi, x_{1}}\right)-a\left(x_{1}, y, \xi+D v^{\xi, x_{2}}\right), D \phi\right) d y \\
& =\int_{Y}\left(a\left(x_{2}, y, \xi+D v^{\xi, x_{2}}\right)-a\left(x_{1}, y, \xi+D v^{\xi, x_{2}}\right), D \phi\right) d y,
\end{aligned}
$$

for every $\phi \in H_{\square}^{1,2}(\Omega)$. In particular for $\phi=D v^{\xi, x_{1}}-D v^{\xi, x_{2}}$ we have

$$
\begin{gathered}
\int_{Y}\left(a\left(x_{1}, y, \xi+D v^{\xi, x_{1}}\right)-a\left(x_{1}, y, \xi+D v^{\xi, x_{2}}\right), \xi+D v^{\xi, x_{1}}-\left(\xi+D v^{\xi, x_{2}}\right)\right) d y \\
=\int_{Y}\left(a\left(x_{2}, y, \xi+D v^{\xi, x_{2}}\right)-a\left(x_{1}, y, \xi+D v^{\xi, x_{2}}\right), D v^{\xi, x_{1}}-D v^{\xi, x_{2}}\right) d y .
\end{gathered}
$$

By applying (5) on the left hand side and the Schwarz and Hölder inequalities on the right hand side it follows that

$$
\begin{aligned}
& \alpha\left(\int_{Y}\left|D v^{\xi, x_{1}}-D v^{\xi, x_{2}}\right|^{2} d y\right)^{\frac{1}{2}} \\
& \quad \leq\left(\int_{Y}\left|a\left(x_{2}, y, \xi+D v^{\xi, x_{2}}\right)-a\left(x_{1}, y, \xi+D v^{\xi, x_{2}}\right)\right|^{2} d y\right)^{\frac{1}{2}} .
\end{aligned}
$$


The continuity condition (10) and (29) imply that

$$
\begin{aligned}
& \alpha\left(\int_{Y}\left|D v^{\xi, x_{1}}-D v^{\xi, x_{2}}\right|^{2} d y\right)^{\frac{1}{2}} \\
& \quad \leq\left(\omega\left(\left|x_{1}-x_{2}\right|\right) \int_{Y}\left|\xi+D v^{\xi, x_{2}}\right|^{2} d y\right)^{\frac{1}{2}} \leq \omega^{\frac{1}{2}}\left(\left|x_{1}-x_{2}\right|\right) \frac{\beta}{\alpha}|\xi| .
\end{aligned}
$$

The result follows by taking (27), (28) and (29) into account.

(b) Let $\xi_{j} \in R^{n}, j=1,2$ and define for a.e. $y \in R^{n}$,

$$
w_{h}^{\xi_{j}, x}(y)=\left(\xi_{j}, y\right)+\varepsilon_{h} v^{\xi_{j}, x}\left(\frac{y}{\varepsilon_{h}}\right)
$$

By the periodicity of $v^{\xi_{j}, x}$ we have that

$$
\begin{aligned}
& w_{h}^{\xi_{j}, x} \rightarrow\left(\xi_{j}, y\right) \quad \text { weakly in } H^{1,2}(\Omega), \\
& D w_{h}^{\xi_{j}, x} \rightarrow \xi_{j} \quad \text { weakly in } L^{2}\left(\Omega, R^{n}\right), \\
& a\left(x, \frac{y}{\varepsilon_{h}}, D w_{h}^{\xi_{j}, x}(y)\right) \rightarrow b\left(x, \xi_{j}\right) \quad \text { weakly in } L^{2}\left(\Omega, R^{n}\right) .
\end{aligned}
$$

The monotonicity condition (5) on $a$ implies that

$$
\begin{gathered}
\int_{\Omega}\left(a\left(x, \frac{y}{\varepsilon_{h}}, D w_{h}^{\xi_{1}, x}\right)-a\left(x, \frac{y}{\varepsilon_{h}}, D w_{h}^{\xi_{2}, x}\right), D w_{h}^{\xi_{1}, x}-D w_{h}^{\xi_{2}, x}\right) \phi(y) d y \\
\geq \alpha \int_{\Omega}\left|D w_{h}^{\xi_{1}, x}-D w_{h}^{\xi_{2}, x}\right|^{2} \phi(y) d y
\end{gathered}
$$

for every $\phi \in C_{0}^{\infty}(\Omega)$ such that $\phi \geq 0$. We apply $\lim _{h \rightarrow \infty} \inf _{h \rightarrow \infty}$ on both sides of this inequality and obtain

$$
\int_{\Omega}\left(b\left(x, \xi_{1}\right)-b\left(x, \xi_{2}\right), \xi_{1}-\xi_{2}\right) \phi(y) d y \geq \alpha \int_{\Omega}\left|\xi_{1}-\xi_{2}\right|^{2} \phi(y) d y
$$

for every $\phi \in C_{0}^{\infty}(\Omega)$, where we on the left hand side have used (31), (32), (8) and Lemma 1 and on the right hand side we have used (31) and the fact that for a weakly convergent sequence $\left(x_{n}\right)$ converging to $x$ we have that $\|x\| \leq \lim \inf _{n \rightarrow \infty}\left\|x_{n}\right\|$. This implies that

$$
\left(b_{i}\left(\xi_{1}\right)-b_{i}\left(\xi_{2}\right), \xi_{1}-\xi_{2}\right) \geq \alpha\left|\xi_{1}-\xi_{2}\right|^{2}
$$

Since $\xi_{1}, \xi_{2}$ were chosen arbitrary (b) is proved. 
(c) Fix $\xi_{1}, \xi_{2} \in R^{n}$. According to (6), Jensen's inequality and (5) we have that

$$
\begin{aligned}
\mid b\left(x, \xi_{1}\right) & -\left.b\left(x, \xi_{2}\right)\right|^{2}=\left|\int_{Y} a\left(x, y, \xi_{1}+D v^{\xi_{1}, x}(y)\right) d y-\int_{Y} a\left(x, y, \xi_{2}+D v^{\xi_{2}, x}(y)\right) d y\right|^{2} \\
\leq & \left(\int_{Y}\left|a\left(x, y, \xi_{1}+D v^{\xi_{1}, x}(y)\right)-a\left(x, y, \xi_{2}+D v^{\xi_{2}, x}(y)\right)\right| d y\right)^{2} \\
\leq & \left(\int_{Y} \beta\left|\xi_{1}+D v^{\xi_{1}, x}(y)-\xi_{2}-D v^{\xi_{2}, x}(y)\right| d y\right)^{2} \\
& \leq \beta^{2} \int_{Y}\left|\xi_{1}+D v^{\xi_{1}, x}(y)-\xi_{2}-D v^{\xi_{2}, x}(y)\right|^{2} d y \\
& \leq \frac{\beta^{2}}{\alpha} \int_{Y}\left(a\left(x, y, \xi_{1}+D v^{\xi_{1}, x}(y)\right)-a\left(x, y, \xi_{2}+D v^{\xi_{2}, x}(y)\right),\right. \\
& \left.\left(\xi_{1}+D v^{\xi_{1}, x}(y)\right)-\left(\xi_{2}+D v^{\xi_{2}, x}(y)\right)\right) d y .
\end{aligned}
$$

Moreover, from the cell problem (16) it follows that

$$
\int_{Y}\left(a\left(x, y, \xi_{k}+D v^{\xi_{k}, x}(y)\right), D v^{\xi_{l}, x}(y)\right) d x=0 \quad \text { for } \quad k, l=1,2,
$$

and we conclude

$$
\begin{aligned}
& \left|b\left(x, \xi_{1}\right)-b\left(x, \xi_{2}\right)\right|^{2} \\
& \quad \leq \frac{\beta^{2}}{\alpha}\left(b\left(x, \xi_{1}\right)-b\left(x, \xi_{2}\right), \xi_{1}-\xi_{2}\right) \leq \frac{\beta^{2}}{\alpha}\left|b\left(x, \xi_{1}\right)-b\left(x, \xi_{2}\right)\right|\left|\xi_{1}-\xi_{2}\right|
\end{aligned}
$$

which implies (c).

(d) Since $a(x, y, 0)=0$ we have that the solution of the cell-problem (16) corresponding to $\xi=0$ is $v^{0, x}=0$. This implies that

$$
b(x, 0)=\int_{Y} a(x, y, 0) d y=0 .
$$

Remark 1. By similar arguments it follows that (b), (c) and (d) holds, up to boundaries, for the homogenized operator $b$ in Theorem 1 .

\section{Some corrector results}

We have proved in both Theorem 1 and Theorem 2 that we for the corresponding solutions have that $u_{u}-u$ converges to 0 weakly in $H_{0}^{1,2}(\Omega)$. By the Rellich imbedding theorem we have that $u_{h}-u$ converges to 0 in $L^{2}(\Omega)$. In general we do not have strong convergence of $D u_{h}-D u$ to 0 in $L^{2}\left(\Omega, R^{n}\right)$. However, we will prove that it is possible to express $D u_{h}$ in terms of $D u$, up to a rest which converges to 0 in $L^{2}\left(\Omega, R^{n}\right)$.

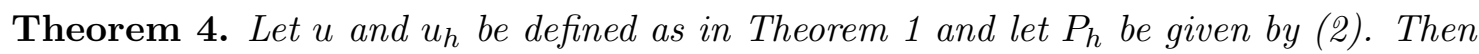

$$
D u_{h}-\sum_{i=1}^{N} \chi_{\Omega_{i}}(x) P_{h}\left(x, M_{h} D u, x_{i}\right) \rightarrow 0 \quad \text { in } \quad L^{2}\left(\Omega, R^{n}\right) .
$$


Proof. In [5] the case $N=1$ was considered. By using these ideas and make the necessary adjustments the proof follows. For the details the reader is referred to [12].

Theorem 5. Let $u$ and $u_{h}$ be defined as in Theorem 2. Moreover, let $P_{h}$ be given by (可) and $\gamma_{h}$ by (因). Then

$$
D u_{h}-P_{h}\left(x, M_{h} D u, \gamma_{h}\right) \rightarrow 0 \quad \text { in } \quad L^{2}\left(\Omega, R^{n}\right)
$$

Proof. We have that

$$
\begin{aligned}
& \left\|D u_{h}-M_{h} D u-D v^{M_{h} D u, \gamma_{h}}\left(\frac{x}{\varepsilon_{h}}\right)\right\|_{L^{2}\left(\Omega, R^{n}\right)} \leq\left\|D u_{h}-D u_{h}^{k}\right\|_{L^{2}\left(\Omega, R^{n}\right)} \\
& +\left\|D u_{h}^{k}-M_{h} D u_{*}^{k}-\sum_{i \in I_{k}} \chi_{\Omega_{i}^{k}}(x) D v^{M_{h} D u_{*}^{k}, x_{i}^{k}}\left(\frac{x}{\varepsilon_{h}}\right)\right\|_{L^{2}\left(\Omega, R^{n}\right)} \\
& +\left\|M_{h} D u_{*}^{k}+\sum_{i \in I_{k}} \chi_{\Omega_{i}^{k}} D v^{M_{h} D u_{*}^{k}, x_{i}^{k}}\left(\frac{x}{\varepsilon_{h}}\right)-M_{h} D u-D v^{M_{h} D u, \gamma_{h}}\left(\frac{x}{\varepsilon_{h}}\right)\right\|_{L^{2}\left(\Omega, R^{n}\right)} .
\end{aligned}
$$

As in the proof of Theorem 2 we have that

$$
\lim _{k \rightarrow \infty} \lim _{h \rightarrow \infty}\left\|D u_{h}-D u_{h}^{k}\right\|_{L^{2}\left(\Omega, R^{n}\right)}=0
$$

and by Theorem 4 it yields that

$$
\lim _{k \rightarrow \infty} \lim _{h \rightarrow \infty}\left\|D u_{h}^{k}-M_{h} D u_{*}^{k}-\sum_{i \in I_{k}} \chi_{\Omega_{i}^{k}}(x) D v^{M_{h} D u_{*}^{k}, x_{i}^{k}}\left(\frac{x}{\varepsilon_{h}}\right)\right\|_{L^{2}\left(\Omega, R^{n}\right)}=0 .
$$

This means that the theorem would be proved if we show that $\lim _{k \rightarrow \infty} \lim _{h \rightarrow \infty}$ acting on the last term in (33) is equal to 0 . In order to prove this fact we make the following elementary estimations:

$$
\begin{aligned}
& \| M_{h} D u_{*}^{k}+\sum_{i \in I_{k}} \chi_{\Omega_{i}^{k}}(x) D v^{M_{h} D u_{*}^{k}, x_{i}^{k}}\left(\frac{x}{\varepsilon_{h}}\right)-M_{h} D u-\left.D v^{M_{h} D u, \gamma_{h}}\left(\frac{x}{\varepsilon_{h}}\right)\right|_{L^{2}\left(\Omega, R^{n}\right)} ^{2} \\
& =\sum_{i \in I_{k}} \int_{\Omega_{i}^{k}}\left|M_{h} D u_{*}^{k}+D v^{M_{h} D u_{*}^{k}, x_{i}^{k}}\left(\frac{x}{\varepsilon_{h}}\right)-M_{h} D u-D v^{M_{h} D u, \gamma_{h}}\left(\frac{x}{\varepsilon_{h}}\right)\right|^{2} d x \\
& \leq \sum_{i \in I_{k}} \int_{\Omega_{i}^{k}}\left(\left|M_{h} D u_{*}^{k}+D v^{M_{h} D u_{*}^{k}, x_{i}^{k}}\left(\frac{x}{\varepsilon_{h}}\right)-M_{h} D u-D v^{M_{h} D u, x_{i}^{k}}\left(\frac{x}{\varepsilon_{h}}\right)\right|\right. \\
& \left.\quad+\left|D v^{M_{h} D u, x_{i}^{k}}\left(\frac{x}{\varepsilon_{h}}\right)-D v^{M_{h} D u, \gamma_{h}}\left(\frac{x}{\varepsilon_{h}}\right)\right|\right)^{2} d x \\
& \leq \sum_{i \in I_{k}} 2 \int_{\Omega_{i}^{k}}\left|M_{h} D u_{*}^{k}+D v^{M_{h} D u_{*}^{k}, x_{i}^{k}}\left(\frac{x}{\varepsilon_{h}}\right)-M_{h} D u-D v^{M_{h} D u, x_{i}^{k}}\left(\frac{x}{\varepsilon_{h}}\right)\right|^{2} d x \\
& \quad+\sum_{i \in I_{k}} 2 \int_{\Omega_{i}^{k}}\left|D v^{M_{h} D u, x_{i}^{k}}\left(\frac{x}{\varepsilon_{h}}\right)-D v^{M_{h} D u, \gamma_{h}}\left(\frac{x}{\varepsilon_{h}}\right)\right|^{2} d x .
\end{aligned}
$$


We shall now study the two terms on the right hand side of (34) separately but first we define,

$$
\xi_{h, *}^{j, k}=\frac{1}{\left|Y_{h}^{j}\right|} \int_{Y_{h}^{j}} D u_{*}^{k} d x
$$

By using (28) and a change of variables we find that we for the first term in (34) have the following estimate

$$
\begin{aligned}
& \int_{\Omega_{i}^{k}}\left|M_{h} D u_{*}^{k}+D v^{M_{h} D u_{*}^{k}, x_{i}^{k}}\left(\frac{x}{\varepsilon_{h}}\right)-M_{h} D u-D v^{M_{h} D u, x_{i}^{k}}\left(\frac{x}{\varepsilon_{h}}\right)\right|^{2} d x \\
& \leq \sum_{j \in J_{h}^{i, k}} \int_{Y_{h}^{j}}\left|\xi_{h, *}^{j, k}+D v^{\xi_{h, *}^{j, k}, x_{i}^{k}}\left(\frac{x}{\varepsilon_{h}}\right)-\xi_{h}^{j}-D v^{\xi_{h}^{j}, x_{i}^{k}}\left(\frac{x}{\varepsilon_{h}}\right)\right|^{2} d x \\
& \quad+\sum_{j \in B_{h}^{i, k}} \int_{Y_{h}^{j}}\left|\xi_{h, *}^{j, k}+D v^{\xi_{h, *}^{j, k}, x_{i}^{k}}\left(\frac{x}{\varepsilon_{h}}\right)-\xi_{h}^{j}-D v^{\xi_{h}^{j}, x_{i}^{k}}\left(\frac{x}{\varepsilon_{h}}\right)\right|^{2} d x \\
& \leq \sum_{j \in J_{h}^{i, k}}\left(\frac{\beta}{\alpha}\right)^{2}\left|\xi_{h, *}^{j, k}-\xi_{h}^{j}\right|^{2}\left|Y_{h}^{j}\right|+\sum_{j \in B_{h}^{i, k}}\left(\frac{\beta}{\alpha}\right)^{2}\left|\xi_{h, *}^{j, k}-\xi_{h}^{j}\right|^{2}\left|Y_{h}^{j}\right| \\
&=\left(\frac{\beta}{\alpha}\right)^{2} \int_{\Omega_{i}^{k, h}}\left|M_{h} D u_{*}^{k}-M_{h} D u\right|^{2} d x+\left(\frac{\beta}{\alpha}\right)^{2} \int_{F_{i}^{k, h}}\left|M_{h} D u_{*}^{k}-M_{h} D u\right|^{2} d x \\
& \leq\left(\frac{\beta}{\alpha}\right)^{2} \int_{\Omega_{i}^{k}}\left|D u_{*}^{k}-D u\right|^{2} d x+\left(\frac{\beta}{\alpha}\right)^{2} \int_{F_{i}^{k, h}}\left|D u_{*}^{k}-D u\right|^{2} d x,
\end{aligned}
$$

where we used Jensen's inequality in the last step. Moreover, by using (29) and Jensen's inequality, we obtain that

$$
\begin{aligned}
\int_{\Omega_{i}^{k}} \mid & D v^{M_{h} D u, x_{i}^{k}}\left(\frac{x}{\varepsilon_{h}}\right)-\left.D v^{M_{h} D u, \gamma_{h}}\left(\frac{x}{\varepsilon_{h}}\right)\right|^{2} d x \\
\leq & \sum_{j \in J_{h}^{i, k}} \int_{Y_{h}^{j}}\left|D v^{\xi_{h}^{j}, x_{i}^{k}}\left(\frac{x}{\varepsilon_{h}}\right)-D v^{\xi_{h}^{j}, x_{h}^{j}}\left(\frac{x}{\varepsilon_{h}}\right)\right|^{2} d x \\
& +\sum_{j \in B_{h}^{i, k}} \int_{Y_{h}^{j}}\left|D v^{\xi_{h}^{j}, x_{i}^{k}}\left(\frac{x}{\varepsilon_{h}}\right)-D v^{\xi_{h}^{j}, x_{h}^{j}}\left(\frac{x}{\varepsilon_{h}}\right)\right|^{2} d x \\
\leq & \sum_{j \in J_{h}^{i, k}} \omega\left(\frac{1}{k}\right) \frac{\beta^{2}}{\alpha^{4}}\left|\xi_{h}^{j}\right|^{2}\left|Y_{h}^{j}\right|+\sum_{j \in B_{h}^{i, k}} \omega\left(\frac{1}{k}+n \varepsilon_{h}\right) \frac{\beta^{2}}{\alpha^{4}}\left|\xi_{h}^{j}\right|^{2}\left|Y_{h}^{j}\right| \\
= & \omega\left(\frac{1}{k}\right) \frac{\beta^{2}}{\alpha^{4}} \int_{\Omega_{i}^{k, h}}\left|M_{h} D u\right|^{2} d x+\omega\left(\frac{1}{k}+n \varepsilon_{h}\right) \frac{\beta^{2}}{\alpha^{4}} \int_{F_{i}^{k, h}}\left|M_{h} D u\right|^{2} d x \\
\leq & \omega\left(\frac{1}{k}\right) \frac{\beta^{2}}{\alpha^{4}} \int_{\Omega_{i}^{k}}|D u|^{2} d x+\omega\left(\frac{1}{k}+n \varepsilon_{h}\right) \frac{\beta^{2}}{\alpha^{4}} \int_{F_{i}^{k, h}}|D u|^{2} d x .
\end{aligned}
$$


By combining (34), (35) and (36) we have that

$$
\begin{gathered}
\left\|M_{h} D u_{*}^{k}+\sum_{i \in I_{k}} \chi_{\Omega_{i}^{k}} D v^{M_{h} D u_{*}^{k}, x_{i}^{k}}\left(\frac{x}{\varepsilon_{h}}\right)-M_{h} D u-D v^{M_{h} D u, \gamma_{h}}\left(\frac{x}{\varepsilon_{h}}\right)\right\|_{L^{2}\left(\Omega, R^{n}\right)}^{2} \\
\quad \leq 2\left(\frac{\beta}{\alpha}\right)^{2}\left(\int_{\Omega}\left|D u_{*}^{k}-D u\right|^{2} d x+\sum_{i \in I_{k}} \int_{F_{i}^{k, h}}\left|D u_{*}^{k}-D u\right|^{2} d x\right) \\
\quad+2 \omega\left(\frac{1}{k}+n \varepsilon_{h}\right) \frac{\beta^{2}}{\alpha^{4}}\left(\int_{\Omega}|D u|^{2} d x+\sum_{i \in I_{k}} \int_{F_{i}^{k, h}}|D u|^{2} d x\right) .
\end{gathered}
$$

Moreover, by noting that $\left|F_{i}^{k, h}\right| \rightarrow 0$ as $h \rightarrow \infty$ and taking (22) into account, we obtain that

$$
\lim _{k \rightarrow \infty} \lim _{h \rightarrow \infty}\left\|M_{h} D u_{*}^{k}+\sum_{i \in I_{k}} \chi_{\Omega_{i}^{k}} D v^{M_{h} D u_{*}^{k}, x_{i}^{k}}\left(\frac{x}{\varepsilon_{h}}\right)-M_{h} D u-D v^{M_{h} D u, \gamma_{h}}\left(\frac{x}{\varepsilon_{h}}\right)\right\|_{L^{2}\left(\Omega, R^{n}\right)}=0
$$

and the theorem is proved.

\section{Acknowledgment}

The author thank the referee for some advises which have improved the final version of this paper.

\section{References}

[1] Bensoussan A., Lions J. and Papanicolaou G., Asymptotic Analysis for Periodic Structures, North Holland, Amsterdam, 1978.

[2] Braides A., Correctors for the Homogenization of Almost Periodic Monotone Operators, Asymptotic Analysis, 1991, V.5, 47-74.

[3] Braides A., Chiado Piat V. and Defranceschi A., Homogenization of Almost Periodic Monotone Operators, Ann. Inst. Henri Poincare, Anal. Non Lineaire, 1992, V.9, N 4, 399-432.

[4] Chiado Piat V. and Defranceschi A., Homogenization of Monotone Operators, Nonlinear Analysis, Theory, Methods and Applications, 1990, V.14, N 9, 717-732.

[5] Dal Maso G. and Defranceschi A., Correctors for the Homogenization of Monotone Operators, Differential and Integral Equations, 1990, V.3, N 6, 1151-1166.

[6] Defranceschi A., An Introduction to Homogenization and G-Convergence, Lecture notes, school on homogenization. ICTP, Trieste, 1993.

[7] Fusco N. and Moscariello G, On the Homogenization of Quasilinear Divergence Structure Operators, Annali Mat. Pura Appl., 1987, V.146, 1-13.

[8] Jikov V., Kozlov S. and Oleinik O., Homogenization of Differential Operators and Integral Functionals, Springer-Verlag, Berlin-Heidelberg-New York, 1994. 
[9] Meyers N. and Elcrat A., On Non-Linear Elliptic Systems and Quasi Regular Functions, Duke Math. J., 1975, V.42, 121-136.

[10] Persson L-E., Persson L., Svanstedt N. and Wyller J., The Homogenization Method: An Introduction, Studentlitteratur, Lund, 1993.

[11] Royden H., Real Analysis, Macmillan, New York, third edition, 1988.

[12] Wall P., Some Homogenization and Corrector Results for Nonlinear Monotone Operators, Research Report 15, ISSN 1400-4003, Dept. of Math., Luleå University of Technology, 1998.

[13] Zeidler E., Nonlinear Functional Analysis and its Applications, Vol.4., Springer Verlag, New York, 1990.

[14] Zeidler E., Nonlinear Functional Analysis and its Applications, Vol.2/B, Springer Verlag, New York, 1990. 